\title{
Control of volunteer adzuki bean in soybean
}

\author{
Christopher Kramer, Nader Soltani", Darren E. Robinson, Clarence J. Swanton, \\ Peter H. Sikkema
}

University of Guelph, Ridgetown Campus, Ridgetown, Canada; ${ }^{*}$ Corresponding Author: nsoltani@ridgetownc.uoguelph.ca

Received 19 March 2012; revised 17 April 2012; accepted 20 May 2012

\section{ABSTRACT}

The objective of this research was to evaluate the efficacy of various pre-emergence (PRE) and post-emergence (POST) herbicides for the control of volunteer adzuki bean (Vigna angularis (Willd.) Ohwi \& Ohashi) in soybean (Glycine max L.). Trials were conducted at two locations in 2005, 2006, 2007, and 2009. Experiments were arranged in a randomized complete block design with either five PRE or nine POST herbicides. Volunteer adzuki bean interference in soybean resulted in yield loss of up to $25 \%$. Cloransulam-methyl, linuron, metribuzin, flumetsulam, and imazethapyr applied PRE provided up to $6 \%$, $24 \%, 14 \%, 8 \%$, and $0 \%$ control, respectively at 8 weeks after emergence (WAE), while acifluorfen, fomesafen, bentazon, thifensulfuron-methyl, cloransulam-methyl, imazethapyr, and imazethapyr plus bentazon applied POST provided $2 \%, 2 \%$, $5 \%, 34 \%, 6 \%, 4 \%$, and $12 \%$ control, respectively at 8 weeks after application (WAA). Generally, with the aforementioned herbicides, soybean yield was equivalent to the weedy control and soybean grain contamination with adzuki bean seed was consistently above the $1 \%$ maximum threshold. Chlorimuron-ethyl and glyphosate applied POST provided up to $84 \%$ and $94 \%$ visual control at 8 WAA, respectively, decreased adzuki bean density, biomass, and seed production, and generally decreased soybean contamination with adzuki bean below the $1 \%$ threshold. The only herbicides evaluated in this study that controlled volunteer adzuki bean in soybean were chlorimuron-ethyl (9 $\mathrm{g}$ ai.ha ${ }^{-1}$ ) and glyphosate $\left(900 \mathrm{~g}\right.$ ai.ha $\left.{ }^{-1}\right)$ applied POST. All the other PRE and POST herbicides evaluated did not provide adequate control of volunteer adzuki bean in soybean.

Keywords: Acifluorfen; Bentazon; Chlorimuron-Ethyl; Cloransulam-Methyl;
Flumetsulam; Fomesafen; Imazethapyr; Linuron; Metribuzin; Pre-Emergence; Post-Emergence; Thifensulfuron-Methyl

\section{INTRODUCTION}

Production of adzuki bean (Vigna angularis (Willd.) Ohwi \& Ohashi) in Ontario has grown over the last few years, and may continue to expand. From only $18 \mathrm{MT}$ in 1994 [1], exports to Japan have reached approximately 5500 MT in 2005, with an additional 2500 MT exported to Taiwan [2]. Estimated land planted to adzuki bean in Ontario has ranged from 2500 to 4000 hectares between 2005 and 2008, and total production ranged from 4500 to 8500 MT [2]. While China exports a large amount of adzuki bean paste to Japan, Ontario has a $65 \%$ market share of global exports of unprocessed adzuki bean to Japan and Taiwan, with the USA and Australia providing the balance [2]. Adzuki bean produced in Ontario, Australia, and the USA is typically of higher quality than that of other locations [1]. Significant importers of adzuki bean include Taiwan and Korea, but Japan is the largest market for adzuki bean since there typically is a gap of 40,000 MT between annual consumption and domestic production [1]. Although it is still a minor crop, there is great potential for the adzuki bean acreage to increase in Ontario.

Despite the opportunity to produce adzuki bean for export to Japan and Taiwan, a significant obstacle for Ontario growers is that volunteer adzuki bean plants will emerge in subsequent years. Adzuki bean pods shatter easily [3], which leads to significant seed losses prior to and during harvest. The seed coat of adzuki beans is hard in comparison to soybean and other legumes, reducing permeability to water and increasing the longevity of seed viability in the soil [4]. Anecdotally, growers have remarked that the presence of volunteer adzuki bean plants can still be an issue even 10 years after the last crop was grown. The actual potential for adzuki bean seed to remain viable in agricultural situations has not yet been documented, although recent laboratory studies have demonstrated that the seeds remain viable for at 
least 5 years at $13 \%$ moisture and $15 \%$ relative humidity [5]. The presence of volunteer adzuki bean plants can be especially problematic where an Identity Preserved (IP) soybean (Glycine max L.) crop is grown. Adzuki bean seeds, at $6 \mathrm{~mm}$ long and $5 \mathrm{~mm}$ wide, are slightly smaller than those of soybean [6]. Consequently, when the soybean crop is harvested, any volunteer adzuki bean seed present may also be harvested and contaminate the grain. IP soybeans containing more than $1 \%$ adzuki bean seed are downgraded and the premium associated with the IP soybeans is lost, severely reducing or eliminating potential profit. Therefore, it is necessary to control volunteer adzuki beans to prevent substantial monetary losses.

In addition to the aforementioned problem, early weed control is essential for optimizing soybean yield. For example, Barrentine [7] concluded soybean seed yield can be reduced by $10 \%, 28 \%, 43 \%$, and $52 \%$ in the presence of Xanthium strumarium L. (common cocklebur) at densities of 3300,6600, 13,000, and 26,000 plants ha ${ }^{-1}$, respectively. A study conducted by Weaver [8] evaluated the effects Chenopodium album L. (common lamb'squarters), Ambrosia artemesiifolia L. (common ragweed), and Setaria spp. (green foxtail) on the yield of soybean. Soybean yield was reduced up to $75 \%, 70 \%$, and $80 \%$ by high densities of Chenopodium album, Ambrosia artemesiifolia, and Setaria spp., respectively [8]. Abutilon theophrasti Medicus (velvetleaf), at populations from 130 to 204 plants $\mathrm{m}^{2}$, has been shown to reduce soybean yields by $23 \%$ [9]. Adzuki bean is not the first volunteer crop issue in soybean. Volunteer maize (Zea mays L.) within a soybean crop is one of the most common volunteer crop problems. Andersen et al. [10] showed that volunteer maize clumps, at the low density of 0.4 clumps per meter of row, decreased soybean yield by $31 \%$. Additionally, Beckett and Stoller [11] reported $21 \%$ and $51 \%$ soybean yield loss at volunteer maize densities of 5380 and 10,760 clumps per hectare, respectively. These studies confirm the requirement of proper weed management to protect crop yield, whether the target species is a traditional weed or a volunteer crop.

The purpose of this study was to evaluate the efficacy of various pre-emergence (PRE) and post-emergence (POST) broadleaf herbicides registered for use in Ontario for the control of volunteer adzuki bean in soybean.

\section{MATERIALS AND METHODS}

Field trials were conducted during the 2005, 2006, 2007 and 2009 growing seasons at Ridgetown and Exeter, Ontario. Sites were moldboard plowed in the autumn, and cultivated in the spring prior to planting. Phosphorus and potash were applied at $112 \mathrm{~kg} \cdot \mathrm{ha}^{-1}$ each prior to plowing in the autumn. Adzuki bean seeds were spread at $55 \mathrm{~kg} \cdot \mathrm{ha}^{-1}$ on the soil surface of the experimental area prior to cultivation in the spring. Soybeans were planted during the month of May of each year. Soil texture, soil organic matter content, soil $\mathrm{pH}$, soybean cultivar, planting date, planting population and herbicide application dates are presented in Table 1.

Two trials were established at each location in a randomized complete block design with four replications. The first experiment at each location evaluated five PRE herbicides: cloransulam-methyl $\left(35 \mathrm{~g}\right.$ ai.ha $\left.{ }^{-1}\right)$, linuron $\left(2250 \mathrm{~g}\right.$ ai.ha $\left.{ }^{-1}\right)$, metribuzin $\left(1120 \mathrm{~g}\right.$ ai.ha $\left.{ }^{-1}\right)$, flumetsulam $\left(70 \mathrm{~g}_{\text {ai.ha }}{ }^{-1}\right)$, and imazethapyr $\left(100 \mathrm{~g}\right.$ ai.ha $\left.{ }^{-1}\right)$, while the second experiment evaluated nine POST herbicides: acifluorfen $\left(600 \mathrm{~g}_{\text {ai.ha }}{ }^{-1}\right)$, fomesafen $\left(240 \mathrm{~g}\right.$ ai.ha $\left.{ }^{-1}\right)$, bentazon $\left(1080 \mathrm{~g}\right.$ ai.ha $\left.{ }^{-1}\right)$, thifensulfuron-methyl $\left(6 \mathrm{~g}\right.$ ai.ha $\left.{ }^{-1}\right)$, chlorimuron-ethyl $\left(9 \mathrm{~g}\right.$ ai.ha $\left.{ }^{-1}\right)$, cloransulam-methyl (17.5 g ai.ha $\left.{ }^{-1}\right)$, imazethapyr (100 $\left.\mathrm{g}^{-1} \cdot \mathrm{ha}^{-1}\right)$, imazethapyr plus bentazon (75 and $840 \mathrm{~g}^{2}$ ai.ha $\left.{ }^{-1}\right)$, and glyphosate $(900 \mathrm{~g}$ ae.ha ${ }^{-1}$ ). Adjuvants used for POST herbicides are listed in Table 5. Each plot consisted of four soybean rows spaced $75 \mathrm{~cm}$ apart and $8.0 \mathrm{~m}$ (Ridgetown) or $10.0 \mathrm{~m}$ (Exeter) in length. The herbicide doses used are the fulllabel dose registered for use in Ontario. Each trial also included two weedy and one weed-free control. The weedfree control was maintained weed-free with an application of glyphosate $\left(1800 \mathrm{~g}\right.$ ae.ha $\left.{ }^{-1}\right)$ applied POST followed by hand hoeing as required.

Herbicide treatments in Ridgetown were applied with a $\mathrm{CO}_{2}$-pressurized backpack sprayer equipped with ULD

Table 1. Environment, soil, soybean information, and herbicide application dates for all experiments.

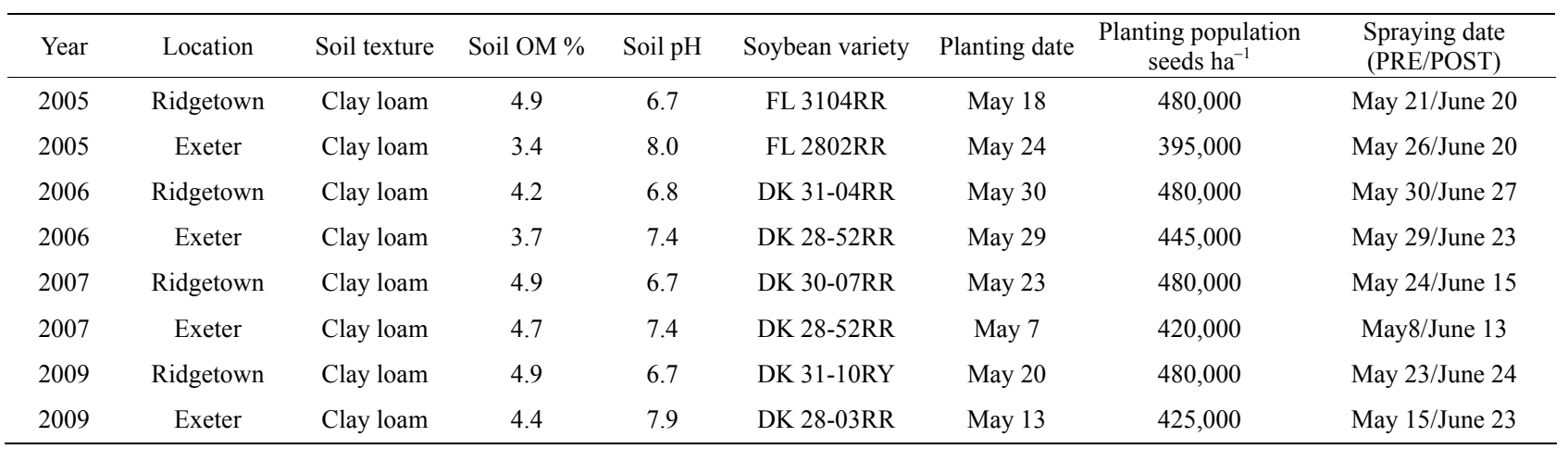


120-02 flat-fan nozzles (Hypro, New Brighton, MN) calibrated to deliver $200 \mathrm{~L} \cdot \mathrm{ha}^{-1}$ of water at $210 \mathrm{kPa}$. In Exeter, herbicide treatments were applied with a $\mathrm{CO}_{2}-$ pressurized backpack sprayer equipped with Teejet 80-02 flat-fan nozzles (Spraying Systems Co., Wheaton, IL) calibrated to deliver $200 \mathrm{~L} \cdot \mathrm{ha}^{-1}$ of water at $241 \mathrm{kPa}$ in 2005, 2006, and 2007, and ULD 120-02 flat-fan nozzles (Hypro, New Brighton, MN) were used in 2009. Herbicide applications were made with a 1.5 meter boom with four nozzles spaced $50 \mathrm{~cm}$ apart over the center two rows.

The PRE herbicides were applied 0 to 3 days after seeding, while the POST herbicides were applied when the adzuki bean was between 5 and $10 \mathrm{~cm}$ in height. Estimate of crop injury was rated 1,2, and 4 weeks after crop emergence (WAE) for the PRE treatments, and 1, 2, and 4 weeks after application (WAA) for the POST treatments. Crop injury was rated visually on a scale of $0 \%$ to $100 \%$, where a rating of 0 was defined as no crop injury and 100 was defined as crop death. At 4 and 8 WAE/ WAA weed control was rated visually on a scale of $0 \%$ to $100 \%$ control, where 0 is no control and 100 is complete volunteer adzuki bean control. At $10 \mathrm{WAE} / \mathrm{WAA}$, adzuki bean density and biomass were determined by counting the number of adzuki bean plants in two $0.25 \mathrm{~m}^{2}$ quadrats in each plot. The adzuki bean plants were cut off at the soil surface, placed in paper bags, dried at $60^{\circ} \mathrm{C}$ to constant weight, and the dry weight was recorded. At crop maturity, the soybeans were harvested from the center two rows with a small-plot combine and the weight and moisture was recorded. Yields were adjusted to $13.5 \%$ moisture. In 2009 at Ridgetown and Exeter, when the volunteer adzuki bean plants within the trials approached maturity, volunteer adzuki bean plants within each plot were removed from two $0.25 \mathrm{~m}^{2}$ quadrats. The number of plants that produced at least one seed pod was recorded, as well as the number of adzuki bean plants that failed to produce pods. The pods were then thrashed and the seeds counted to determine the number of seeds produced by surviving adzuki bean plants in each treatment. Additionally, samples were drawn from the harvested soybean to evaluate the percent contamination. A $100 \mathrm{~g}$ soybean sample was taken, from which the adzuki bean within the sample were removed and weighed to determine the percent contamination. Commercially, soybean samples with greater than $1 \%$ adzuki bean seed would be subject to downgrading and loss of the IP soybean premium.

In 2009, permanent quadrats were established within the weed-free plots at Ridgetown and left undisturbed for the entire growing season to determine the emergence pattern of volunteer adzuki bean within a soybean crop. On a weekly basis the emerged adzuki beans were counted and then physically removed.
An analysis of variance was conducted on all data. Data were analyzed using the MIXED procedure in SAS (Ver. 9.1, SAS Institute INC., Cary, NC). Variances were separated into the random effects of environment (year and location), replication (within environment), and environment by treatment interaction, and the fixed effect of herbicide treatment. Significance of random effects and their interaction with fixed effects was tested using the Z-test of the variance estimate, while the significance of fixed effects was tested using the F-test. Due to significant environment by treatment interactions resulting from varied levels of control by certain herbicides over years and location, the pooling of data was restricted to combinations of certain environments and is presented accordingly. To ensure the assumptions of the variance analysis (errors are independent, homogeneous, and normally distributed) were met, residuals were plotted by predicted, treatment, and replication for each variable analyzed. In addition, data were tested for normality using the Shapiro-Wilk statistic as generated by the PROC UNIVARIATE procedure in SAS. When necessary to achieve normality, data transformations (natural log transformation, square root transformation, and arcsine square root transformation) were applied and chosen by that transformation which produced the highest Shapiro-Wilk statistic. Treatment means were transformed back to the original scale for presentation. Means were separated using Fisher's protected LSD at $P<0.05$.

\section{RESULTS AND DISCUSSION}

\subsection{Pre-Emergence Herbicides}

The PRE herbicides evaluated did not cause commercially significant injury to soybean. At 4 WAE, crop injury was less than $2 \%$ for all herbicides (data not presented).

The PRE herbicides evaluated did not provide acceptable control of volunteer adzuki bean. Cloransulammethyl (35 $\mathrm{g}$ ai.ha $\left.{ }^{-1}\right)$, linuron (2250 $\mathrm{g}$ ai.ha $\left.{ }^{-1}\right)$, metribuzin (1120 g ai.ha $\left.{ }^{-1}\right)$, flumetsulam $\left(70 \mathrm{~g}\right.$ ai.ha $\left.{ }^{-1}\right)$, and imazethapyr $\left(100 \mathrm{~g} \mathrm{ai}^{-1} \mathrm{ha}^{-1}\right)$ provided up to $8 \%, 44 \%, 21 \%$, $18 \%$, and $2 \%$ control 4 WAE (Table 2) and up to $6 \%$, $24 \%, 14 \%, 8 \%$, and $0 \%$ control 8 WAE, respectively (Table 3). This is consistent with research in Phaseolus vulgaris, where Soltani et al. [12] found linuron injured black bean $(27 \%)$ and white bean $(31 \%)$ at 4 WAE. In addition, a study evaluating flumetsulam use in southern pea (Vigna unguiculata L.) reported that there was crop injury of $13 \%$ at 3 WAA, demonstrating southern pea's relative tolerance to flumetsulam [13]. Harrison [14] concluded that adzuki bean is tolerant to metribuzin, while Soltani et al. [15] showed that imazethapyr at 150 $\mathrm{g}$ ai.ha ${ }^{-1}$ applied preplant incorporated had no effect on adzuki bean yield or maturity and is suitable for in crop 
Table 2. Means for percent volunteer adzuki bean control in soybean with various PRE herbicides 4 WAE at Ridgetown and Exeter, ON from 2005 to $2009^{\mathrm{a}}$.

\begin{tabular}{|c|c|c|c|c|c|}
\hline & Dose $\mathrm{g}$ ai.ha ${ }^{-1}$ & 2005 & 2006 & $2007^{\mathrm{b}}$ & 2009 \\
\hline Untreated control & & $0 \mathrm{~b}$ & $0 \mathrm{c}$ & $0 \mathrm{~d}$ & $0 \mathrm{~b}$ \\
\hline Weed free control & & $100 \mathrm{a}$ & $100 \mathrm{a}$ & $100 \mathrm{a}$ & $100 \mathrm{a}$ \\
\hline Cloransulam-methyl & 35 & $4 \mathrm{~b}$ & $0 \mathrm{c}$ & $8 \mathrm{c}$ & $7 \mathrm{~b}$ \\
\hline Linuron & 2250 & $14 \mathrm{~b}$ & $2 \mathrm{bc}$ & $44 \mathrm{~b}$ & $25 \mathrm{~b}$ \\
\hline Metribuzin & 1120 & $8 \mathrm{~b}$ & $4 \mathrm{~b}$ & $21 \mathrm{c}$ & $17 \mathrm{~b}$ \\
\hline Flumetsulam & 70 & $5 \mathrm{~b}$ & $7 \mathrm{~b}$ & $18 \mathrm{c}$ & $9 \mathrm{~b}$ \\
\hline Imazethapyr & 100 & $0 \mathrm{~b}$ & $2 \mathrm{bc}$ & $0 \mathrm{~d}$ & $0 \mathrm{~b}$ \\
\hline SE & & 5 & 5 & 7 & 6 \\
\hline
\end{tabular}

${ }^{a}$ Means followed by the same letter within a column are not significantly different according to Fisher's Protected LSD at $P<0.05$; ${ }^{\text {b } 2007 ~ d a t a ~ f r o m ~ R i d g e t o w n ~}$ experiment only.

Table 3. Means for percent volunteer adzuki bean control in soybean with various PRE herbicides 8 WAE, density and biomass 10 WAE, and soybean yield at crop maturity at Exeter and Ridgetown, ON from 2005 to $2009^{\mathrm{a}}$.

\begin{tabular}{|c|c|c|c|c|c|c|c|c|}
\hline \multirow[b]{2}{*}{ Treatments } & \multirow{2}{*}{$\begin{array}{c}\text { Dose } \\
\text { g ai.ha }^{-1}\end{array}$} & \multirow{2}{*}{$\begin{array}{c}2005 / 2006 \\
\% \\
\end{array}$} & \multirow{2}{*}{$\begin{array}{c}2007^{\mathrm{b}} \\
\%\end{array}$} & \multirow{2}{*}{$\begin{array}{c}2009 \\
\%\end{array}$} & \multirow{2}{*}{$\begin{array}{c}\text { Density } \\
\#^{2}{ }^{2}\end{array}$} & \multirow{2}{*}{$\begin{array}{c}\text { Biomass } \\
\mathrm{g} \mathrm{m}^{2}\end{array}$} & \multicolumn{2}{|c|}{ Yield T.ha ${ }^{-1}$} \\
\hline & & & & & & & Exeter & Ridgetown \\
\hline Untreated control & & $0.0 \mathrm{~d}$ & $0 \mathrm{~d}$ & $0 \mathrm{~b}$ & $73 \mathrm{~cd}$ & $128 \mathrm{~d}$ & $2.45 \mathrm{~b}$ & $2.54 \mathrm{c}$ \\
\hline Weed free control & & $100.0 \mathrm{a}$ & $100 \mathrm{a}$ & $100 \mathrm{a}$ & $0 \mathrm{a}$ & $0 \mathrm{a}$ & $3.11 \mathrm{a}$ & $3.33 \mathrm{a}$ \\
\hline Cloransulam-methyl & 35 & $0.6 \mathrm{bcd}$ & $6 \mathrm{~cd}$ & $6 \mathrm{~b}$ & $64 \mathrm{bcd}$ & $119 \mathrm{bcd}$ & $2.42 \mathrm{~b}$ & $2.71 \mathrm{bc}$ \\
\hline Linuron & 2250 & $1.4 \mathrm{~b}$ & $24 \mathrm{~b}$ & $22 \mathrm{~b}$ & $52 \mathrm{~b}$ & $97 \mathrm{~b}$ & $2.62 \mathrm{~b}$ & $2.74 \mathrm{bc}$ \\
\hline Metribuzin & 1120 & $0.5 \mathrm{bcd}$ & $5 \mathrm{~cd}$ & $14 \mathrm{~b}$ & $60 \mathrm{bc}$ & $105 \mathrm{bc}$ & $2.62 \mathrm{~b}$ & $2.83 \mathrm{~b}$ \\
\hline Flumetsulam & 70 & $1.0 \mathrm{bcd}$ & $8 \mathrm{bc}$ & $8 \mathrm{~b}$ & $63 \mathrm{bc}$ & $127 \mathrm{~cd}$ & $2.45 \mathrm{~b}$ & $2.83 \mathrm{~b}$ \\
\hline Imazethapyr & 100 & $0.3 \mathrm{~cd}$ & $0 \mathrm{~d}$ & $0 \mathrm{~b}$ & $81 \mathrm{~d}$ & $158 \mathrm{e}$ & $2.31 \mathrm{~b}$ & $2.74 \mathrm{bc}$ \\
\hline SE & & 3.8 & 7 & 5 & 3 & 6 & 0.04 & 0.05 \\
\hline
\end{tabular}

${ }^{a}$ Means followed by the same letter within a column are not significantly different according to Fisher's Protected LSD at $P<0.05 ;{ }^{b} 2007$ data from Ridgetown experiment only.

use.

Similar to the control ratings, linuron reduced adzuki bean density and dry weight $29 \%$ and $24 \%$, respectively (Table 3). The remaining PRE herbicides decreased volunteer adzuki bean density and dry weight $18 \%$ or less. There was no effect of the PRE herbicides evaluated on volunteer adzuki bean plants with pods, adzuki bean seed production, or seeds per plant, further indicating the lack of volunteer adzuki bean control with these herbicides (Table 4). Additionally, there was no effect on soybean seed contamination compared to the weedy control. Soybean seed from each of the PRE herbicide treatments exceeded $9 \%$ contamination with adzuki bean seed. PRE application of cloransulam-methyl, linuron, metribuzin, flumetsulam, and imazethapyr did not provide acceptable control of volunteer adzuki bean in soybean.

\subsection{Post-Emergence Herbicides}

The POST herbicides, with the exception of thifensulfuron-methyl, did not cause commercially significant soybean injury (>10\%) at 1, 2 and 4 WAA (Tables 5 and 6).
Thifensulfuron-methyl (6 $\mathrm{g}$ ai.ha ${ }^{-1}$ ) applied POST at Exeter in 2009 resulted in $15 \%$ and $14 \%$ crop injury 1 and 2 WAA, respectively. This injury was transient with only $7 \%$ injury observed 4 WAA (Table 6).

Most of the POST applied herbicides provided poor control of volunteer adzuki bean. Acifluorfen (600 g ai.ha $\left.{ }^{-1}\right)$, fomesafen (240 g ai.ha $\left.{ }^{-1}\right)$, bentazon (1080 g ai.ha $\left.{ }^{-1}\right)$, thifensulfuron-methyl $\left(6 \mathrm{~g}\right.$ ai.ha $\left.{ }^{-1}\right)$, cloransulammethyl (17.5 $\mathrm{g}$ ai.ha $\left.{ }^{-1}\right)$, imazethapyr (100 g ai.ha $\left.{ }^{-1}\right)$, and imazethapyr plus bentazon (75 plus $840 \mathrm{~g}$ ai.ha ${ }^{-1}$ ) applied POST provided up to $12 \%, 20 \%, 24 \%, 43 \%, 23 \%$, $12 \%$, and $20 \%$ control 4 WAA (Table 7) and 2\%, $2 \%$, $5 \%, 34 \%, 6 \%, 4 \%$, and $12 \%$ control 8 WAA, respectively (Table 8). In addition, these herbicides reduced volunteer adzuki bean density and dry weight only $34 \%$ or less (Table 9). Previous research focusing on the tolerance of adzuki bean and other Vigna species to some of the herbicides mentioned above supports the conclusion that these herbicides are inadequate for volunteer adzuki bean control in soybean. Jones et al. [16] demonstrated poor Vigna unguiculata L. (cowpea) control with acifluorfen of $48 \% 4$ WAA, while Murphy and Gossett [17] reported 
Table 4. Means for adzuki bean pod and seed production, and soybean seed contamination with various PRE herbicides at Ridgetown and Exeter, ON from 2005 to 2009 [There was no treatment effect to discern the differences between the herbicide applications and untreated control, therefore, mean separations were not performed].

\begin{tabular}{|c|c|c|c|c|}
\hline Treatments & Dose g ai.ha ${ }^{-1}$ & $\begin{array}{l}\text { Plants with } \\
\text { Pods \# } \text { m }^{2}\end{array}$ & $\begin{array}{l}\text { Seeds } \\
\# \mathrm{~m}^{2}\end{array}$ & $\begin{array}{c}\text { Soybean } \\
\text { contamination \% }\end{array}$ \\
\hline Untreated control & & 51 & 367 & 12 \\
\hline Weed free control & & 0 & 0 & 0 \\
\hline Cloransulam-methyl & 35 & 56 & 422 & 12 \\
\hline Linuron & 2250 & 48 & 275 & 10 \\
\hline Metribuzin & 1120 & 34 & 226 & 9 \\
\hline Flumetsulam & 70 & 51 & 347 & 11 \\
\hline Imazethapyr & 100 & 59 & 484 & 14 \\
\hline Untreated control & & 50 & 405 & 13 \\
\hline
\end{tabular}

Table 5. Means for soybean injury with various POST herbicides 1 WAA at Ridgetown and Exeter, ON from 2005 to $2009^{\mathrm{a}}$.

\begin{tabular}{|c|c|c|c|c|c|c|}
\hline \multirow{2}{*}{ Treatments } & \multirow{2}{*}{$\begin{array}{l}\text { Dose } \\
\text { g ai.ha }\end{array}$} & \multicolumn{2}{|c|}{ Ridgetown } & \multicolumn{3}{|c|}{ Exeter } \\
\hline & & $2005 / 06$ & 2007 & $2005 / 07$ & 2006 & 2009 \\
\hline Untreated control & & $0 \mathrm{a}$ & $0 \mathrm{a}$ & $0 \mathrm{a}$ & $0 \mathrm{a}$ & $0.0 \mathrm{a}$ \\
\hline Weed free control & & $0 \mathrm{a}$ & $0 \mathrm{a}$ & $0 \mathrm{a}$ & $0 \mathrm{a}$ & $0.0 \mathrm{a}$ \\
\hline Acifluorfen & 600 & $3 \mathrm{c}$ & $3 \mathrm{~cd}$ & $1 \mathrm{bc}$ & $0 \mathrm{a}$ & $7.2 \mathrm{~cd}$ \\
\hline Fomesafen $^{\mathrm{b}}$ & 240 & $2 \mathrm{bc}$ & $4 \mathrm{~d}$ & $2 \mathrm{bcd}$ & $4 \mathrm{~b}$ & $7.7 \mathrm{~cd}$ \\
\hline Bentazon & 1080 & $2 \mathrm{bc}$ & $1 \mathrm{ab}$ & $4 \mathrm{~cd}$ & $6 \mathrm{~b}$ & $6.0 \mathrm{c}$ \\
\hline Thifensulfuron-methyl $^{\mathrm{c}}$ & 6 & $2 \mathrm{bc}$ & $5 \mathrm{~d}$ & 2 bcd & $0 \mathrm{a}$ & $15.0 \mathrm{e}$ \\
\hline Chlorimuron-ethyl ${ }^{\text {de }}$ & 9 & $1 \mathrm{bc}$ & $3 \mathrm{~cd}$ & $4 \mathrm{~cd}$ & $6 \mathrm{~b}$ & $9.2 \mathrm{~d}$ \\
\hline Cloransulam-methyl ${ }^{\mathrm{ef}}$ & 17.5 & $1 \mathrm{bc}$ & $2 \mathrm{bc}$ & $1 \mathrm{ab}$ & $0 \mathrm{a}$ & $4.1 \mathrm{~b}$ \\
\hline Imazethapyr $^{\mathrm{ef}}$ & 100 & $2 \mathrm{bc}$ & $3 \mathrm{~cd}$ & $1 \mathrm{ab}$ & $0 \mathrm{a}$ & $9.2 \mathrm{~d}$ \\
\hline 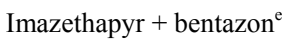 & $75+840$ & $2 \mathrm{bc}$ & $1 \mathrm{ab}$ & $5 \mathrm{~d}$ & $8 \mathrm{c}$ & $6.5 \mathrm{c}$ \\
\hline Glyphosate & 900 & $0 \mathrm{a}$ & $0 \mathrm{a}$ & $0 \mathrm{a}$ & $0 \mathrm{a}$ & $0.0 \mathrm{a}$ \\
\hline SE & & 0.2 & 0.4 & 0.3 & 1 & 0.7 \\
\hline
\end{tabular}

${ }^{\mathrm{a}}$ Means followed by the same letter within a column are not significantly different according to Fisher's Protected LSD at $P<0.05$; ${ }^{\mathrm{b}}$ Included Turbocharge $(0.5 \% \mathrm{v} / \mathrm{v}) ;{ }^{\mathrm{c}}$ Included non-ionic surfactant $(0.1 \% \mathrm{v} / \mathrm{v})$ and UAN $28 \%\left(8.0 \mathrm{~L} \cdot \mathrm{ha}^{-1}\right) ;{ }^{\mathrm{d}}$ Included non-ionic surfactant $(0.2 \% \mathrm{v} / \mathrm{v}) ;{ }^{\mathrm{e}}$ Included UAN $28 \%(2.0$ $\left.\mathrm{L} \cdot \mathrm{ha}^{-1}\right) ;{ }^{\mathrm{f}}$ Included non-ionic surfactant $(0.25 \% \mathrm{v} / \mathrm{v})$.

Table 6. Means for soybean injury with various POST herbicides 2 and 4 WAA at Ridgetown and Exeter, ON from 2005 to $2009^{\mathrm{a}}$.

\begin{tabular}{|c|c|c|c|c|c|c|c|}
\hline \multirow{3}{*}{ Treatments } & \multirow{3}{*}{$\begin{array}{c}\text { Dose } \\
\text { g ai.ha }\end{array}$} & \multicolumn{4}{|c|}{ Injury 2 WAA } & \multirow{2}{*}{\multicolumn{2}{|c|}{$\begin{array}{c}\text { Injury } 4 \text { WAA } \\
\text { Exeter }\end{array}$}} \\
\hline & & \multirow{2}{*}{$\begin{array}{l}\text { Ridgetown } \\
2005 / 06 / 07\end{array}$} & \multicolumn{3}{|c|}{ Exeter } & & \\
\hline & & & $2005 / 06$ & 2007 & 2009 & 2007 & 2009 \\
\hline Untreated control & & $0 \mathrm{a}$ & $0 \mathrm{a}$ & $0 \mathrm{a}$ & $0 \mathrm{a}$ & $0 \mathrm{a}$ & $0 \mathrm{a}$ \\
\hline Weed free control & & $0 \mathrm{a}$ & $0 \mathrm{a}$ & $0 \mathrm{a}$ & $0 \mathrm{a}$ & $0 \mathrm{a}$ & $0 \mathrm{a}$ \\
\hline Acifluorfen & 600 & $1 \mathrm{ab}$ & $0 \mathrm{a}$ & $0 \mathrm{a}$ & $6 \mathrm{~cd}$ & $0 \mathrm{a}$ & $5 \mathrm{c}$ \\
\hline Fomesafen $^{\mathrm{b}}$ & 240 & $1 \mathrm{ab}$ & $1 \mathrm{~b}$ & $0 \mathrm{a}$ & $6 \mathrm{~cd}$ & $0 \mathrm{a}$ & $6 \mathrm{~cd}$ \\
\hline Bentazon & 1080 & $1 \mathrm{ab}$ & $2 \mathrm{c}$ & $0 \mathrm{a}$ & $5 \mathrm{c}$ & $0 \mathrm{a}$ & $2 b$ \\
\hline Thifensulfuron-methyl ${ }^{\mathrm{c}}$ & 6 & $2 \mathrm{~b}$ & $0 \mathrm{a}$ & $1 \mathrm{~b}$ & $14 \mathrm{f}$ & $0 \mathrm{a}$ & $7 d$ \\
\hline Chlorimuron-ethyl $\mathrm{de}^{\mathrm{de}}$ & 9 & $1 \mathrm{ab}$ & $0 \mathrm{a}$ & $0 \mathrm{a}$ & $8 \mathrm{e}$ & $0 \mathrm{a}$ & $3 b$ \\
\hline Cloransulam-methyl ${ }^{\mathrm{ef}}$ & 17.5 & $1 \mathrm{ab}$ & $0 \mathrm{a}$ & $0 \mathrm{a}$ & $2 b$ & $0 \mathrm{a}$ & $2 \mathrm{~b}$ \\
\hline Imazethapyr $^{\mathrm{ef}}$ & 100 & $1 \mathrm{ab}$ & $0 \mathrm{a}$ & $2 \mathrm{~b}$ & $7 \mathrm{de}$ & $4 \mathrm{~b}$ & $9 \mathrm{e}$ \\
\hline 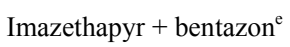 & $75+840$ & $1 \mathrm{ab}$ & $2 \mathrm{c}$ & $2 \mathrm{~b}$ & $5 \mathrm{c}$ & $6 \mathrm{~b}$ & $5 \mathrm{c}$ \\
\hline Glyphosate & 900 & $0 \mathrm{a}$ & $0 \mathrm{a}$ & $0 \mathrm{a}$ & $0 \mathrm{a}$ & $0 \mathrm{a}$ & $0 \mathrm{a}$ \\
\hline SE & & 0.1 & 0.1 & 0.4 & 0.7 & 0.5 & 0.5 \\
\hline
\end{tabular}

${ }^{\mathrm{a}}$ Means followed by the same letter within a column are not significantly different according to Fisher's Protected LSD at $P<0.05$; ${ }^{\mathrm{b}}$ Included Turbocharge $(0.5 \% \mathrm{v} / \mathrm{v}) ;{ }^{\mathrm{c}}$ Included non-ionic surfactant $(0.1 \% \mathrm{v} / \mathrm{v})$ and UAN $28 \%\left(8.0 \mathrm{~L} \cdot \mathrm{ha}^{-1}\right) ;{ }^{\mathrm{d}}$ Included non-ionic surfactant $(0.2 \% \mathrm{v} / \mathrm{v}) ;{ }^{\mathrm{e}}$ ncluded UAN $28 \%(2.0$ $\left.\mathrm{L} \cdot \mathrm{ha}^{-1}\right) ;{ }^{\mathrm{f}}$ Included non-ionic surfactant $(0.25 \% \mathrm{v} / \mathrm{v})$. 
Table 7. Means for percent volunteer adzuki bean control in soybean with various POST herbicides 4 WAA at Ridgetown and Exeter, ON from 2005 to $2009^{\mathrm{a}}$.

\begin{tabular}{|c|c|c|c|c|c|c|c|}
\hline \multirow{2}{*}{ Treatments } & \multirow{2}{*}{$\begin{array}{c}\text { Dose } \\
\text { g ai.ha }\end{array}$} & \multicolumn{2}{|c|}{2005} & \multicolumn{2}{|c|}{$2006 / 07$} & \multicolumn{2}{|c|}{2009} \\
\hline & & Ridgetown & Exeter & Ridgetown & Exeter & Ridgetown & Exeter \\
\hline Untreated control & & $0 \mathrm{f}$ & $0 \mathrm{e}$ & $0 \mathrm{e}$ & $0 \mathrm{~d}$ & $0 \mathrm{f}$ & $0 \mathrm{~g}$ \\
\hline Weed free control & & $100 \mathrm{a}$ & $100 \mathrm{a}$ & $100 \mathrm{a}$ & $100 \mathrm{a}$ & $100 \mathrm{a}$ & $100 \mathrm{a}$ \\
\hline Acifluorfen & 600 & 1 ef & $0 \mathrm{e}$ & $6 \mathrm{de}$ & $1 \mathrm{~d}$ & 4 def & $12 \mathrm{e}$ \\
\hline Fomesafen $^{\mathrm{b}}$ & 240 & $0 \mathrm{f}$ & $0 \mathrm{e}$ & $2 \mathrm{e}$ & $1 \mathrm{~d}$ & $9 \mathrm{~d}$ & 20 de \\
\hline Bentazon & 1080 & $0 \mathrm{f}$ & $24 \mathrm{~d}$ & $15 \mathrm{~d}$ & $18 \mathrm{c}$ & $7 \mathrm{~d}$ & $17 \mathrm{e}$ \\
\hline Thifensulfuron-methyl $^{\mathrm{c}}$ & 6 & $34 \mathrm{c}$ & $25 \mathrm{~d}$ & $43 \mathrm{c}$ & $31 \mathrm{c}$ & $36 \mathrm{c}$ & $32 \mathrm{~d}$ \\
\hline Chlorimuron-ethyl $^{\text {de }}$ & 9 & $90 \mathrm{~b}$ & $45 \mathrm{c}$ & $84 \mathrm{~b}$ & $82 \mathrm{~b}$ & $76 \mathrm{~b}$ & $74 \mathrm{~b}$ \\
\hline Cloransulam-methyl $^{\mathrm{ef}}$ & 17.5 & $3 \mathrm{de}$ & $23 \mathrm{~d}$ & $10 \mathrm{de}$ & $3 \mathrm{~d}$ & 4 def & $15 \mathrm{e}$ \\
\hline Imazethapyr ${ }^{\mathrm{ef}}$ & 100 & $5 \mathrm{~d}$ & $5 \mathrm{e}$ & $12 \mathrm{~d}$ & $2 \mathrm{~d}$ & $5 \mathrm{de}$ & $3 \mathrm{f}$ \\
\hline 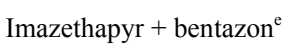 & $75+840$ & $0 \mathrm{f}$ & $25 \mathrm{~d}$ & $15 \mathrm{~d}$ & $16 \mathrm{c}$ & $1 \mathrm{ef}$ & $20 \mathrm{e}$ \\
\hline Glyphosate & 900 & $99 \mathrm{a}$ & $71 \mathrm{~b}$ & $94 \mathrm{~b}$ & $87 \mathrm{~b}$ & $72 b$ & $55 \mathrm{c}$ \\
\hline $\mathrm{SE}$ & & 7 & 5 & 4 & 4 & 6 & 5 \\
\hline
\end{tabular}

${ }^{a}$ Means followed by the same letter within a column are not significantly different according to Fisher's Protected LSD at $P<0.05$; ${ }^{\mathrm{b}}$ Included Turbocharge $(0.5 \% \mathrm{v} / \mathrm{v}) ;{ }^{\mathrm{c}}$ Included non-ionic surfactant $(0.1 \% \mathrm{v} / \mathrm{v})$ and UAN $28 \%\left(8.0 \mathrm{~L} \cdot \mathrm{ha}^{-1}\right) ;{ }^{\mathrm{d}}$ Included non-ionic surfactant $(0.2 \% \mathrm{v} / \mathrm{v}) ;{ }^{\mathrm{e}}$ Included UAN $28 \%\left(2.0 \mathrm{~L} \cdot \mathrm{ha}{ }^{-1}\right) ;$ ${ }^{\mathrm{f}}$ Included non-ionic surfactant $(0.25 \% \mathrm{v} / \mathrm{v})$.

Table 8. Means for percent volunteer adzuki bean control in soybean with various POST herbicides 8 WAA at Ridgetown and Exeter, ON from 2005 to $2009^{\mathrm{a}}$.

\begin{tabular}{|c|c|c|c|c|c|c|}
\hline \multirow{2}{*}{ Treatments } & \multirow{2}{*}{$\begin{array}{c}\text { Dose } \\
\text { g ai.ha }\end{array}$} & \multicolumn{2}{|c|}{ Ridgetown } & \multicolumn{3}{|c|}{ Exeter } \\
\hline & & $2005 / 06 / 07$ & 2009 & $2005 / 2009$ & 2006 & 2007 \\
\hline Untreated control & & $0 \mathrm{f}$ & $0 \mathrm{e}$ & $0 \mathrm{~d}$ & $0 \mathrm{~d}$ & $0 \mathrm{e}$ \\
\hline Weed free control & & $100 \mathrm{a}$ & $100 \mathrm{a}$ & $100 \mathrm{a}$ & $100 \mathrm{a}$ & $100 \mathrm{a}$ \\
\hline Acifluorfen & 600 & $2 \mathrm{ef}$ & $0 \mathrm{e}$ & $2 \mathrm{~cd}$ & $0 \mathrm{~d}$ & $0 \mathrm{e}$ \\
\hline Fomesafen $^{\mathrm{b}}$ & 240 & $0 \mathrm{f}$ & $0 \mathrm{e}$ & $2 \mathrm{~cd}$ & $0 \mathrm{~d}$ & $0 \mathrm{e}$ \\
\hline Bentazon & 1080 & $2 \mathrm{ef}$ & $0 \mathrm{e}$ & $5 \mathrm{~cd}$ & $0 \mathrm{~d}$ & $5 \mathrm{e}$ \\
\hline Thifensulfuron-methyl ${ }^{c}$ & 6 & $21 \mathrm{~d}$ & $15 \mathrm{~d}$ & $13 \mathrm{c}$ & $0 \mathrm{~d}$ & $34 \mathrm{~d}$ \\
\hline Chlorimuron-ethyl ${ }^{\mathrm{de}}$ & 9 & $81 \mathrm{c}$ & $84 \mathrm{~b}$ & $66 \mathrm{~b}$ & $72 \mathrm{c}$ & $74 \mathrm{~b}$ \\
\hline Cloransulam-methyl $^{\mathrm{ef}}$ & 17.5 & $1 \mathrm{ef}$ & $0 \mathrm{e}$ & $2 \mathrm{~cd}$ & $0 \mathrm{~d}$ & $6 \mathrm{e}$ \\
\hline Imazethapyr $^{\mathrm{ef}}$ & 100 & $4 \mathrm{ef}$ & $0 \mathrm{e}$ & $0 \mathrm{~d}$ & $0 \mathrm{~d}$ & $0 \mathrm{e}$ \\
\hline 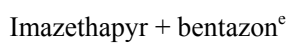 & $75+840$ & $1 \mathrm{ef}$ & $0 \mathrm{e}$ & $12 \mathrm{c}$ & $0 \mathrm{~d}$ & $5 \mathrm{e}$ \\
\hline Glyphosate & 900 & $94 \mathrm{~b}$ & $71 \mathrm{c}$ & $66 \mathrm{~b}$ & $88 \mathrm{~b}$ & $64 \mathrm{c}$ \\
\hline SE & & 4 & 6 & 3 & 6 & 6 \\
\hline
\end{tabular}

${ }^{\mathrm{a}}$ Means followed by the same letter within a column are not significantly different according to Fisher's Protected LSD at $P<0.05 ;{ }^{\mathrm{b}}$ Included Turbocharge $(0.5 \% \mathrm{v} / \mathrm{v}) ;{ }^{\mathrm{c}}$ Included non-ionic surfactant $(0.1 \% \mathrm{v} / \mathrm{v})$ and UAN $28 \%\left(8.0 \mathrm{~L} \cdot \mathrm{ha}^{-1}\right) ;{ }^{\mathrm{d}}$ Included non-ionic surfactant $(0.2 \% \mathrm{v} / \mathrm{v}) ;{ }^{\mathrm{e}} \mathrm{Included} \mathrm{UAN} 28 \%\left(2.0 \mathrm{~L} \cdot \mathrm{ha}{ }^{-1}\right)$; ${ }^{\mathrm{f}}$ Included non-ionic surfactant $(0.25 \% \mathrm{v} / \mathrm{v})$. 
Table 9. Means for volunteer adzuki bean density and biomass in soybean with various POST herbicides 10 WAA at Ridgetown and Exeter, ON from 2005 to $2009^{\mathrm{a}}$.

\begin{tabular}{|c|c|c|c|c|c|c|}
\hline & \multirow[t]{2}{*}{ Dose $g$ ai.ha $^{-1}$} & \multicolumn{3}{|c|}{ Density \# $\mathrm{m}^{2}$} & \multicolumn{2}{|c|}{ Biomass $\mathrm{g} \mathrm{m}^{2}$} \\
\hline & & $2006 / 07$ & $2005 / 09$ & 2009 & $2005 / 07 / 09$ & 2006 \\
\hline Treatments & & & Ridgetown & Exeter & & \\
\hline Untreated control & & $77 \mathrm{~cd}$ & $54 \mathrm{~cd}$ & $83 \mathrm{e}$ & $97 \mathrm{de}$ & $248 \mathrm{e}$ \\
\hline Weed free control & & $0 \mathrm{a}$ & $0 \mathrm{a}$ & $0 \mathrm{a}$ & $0 \mathrm{a}$ & $0 \mathrm{a}$ \\
\hline Acifluorfen & 600 & $76 \mathrm{~cd}$ & $58 \mathrm{~cd}$ & $61 \mathrm{~cd}$ & $88 \mathrm{~cd}$ & $232 \mathrm{de}$ \\
\hline Fomesafen $^{\mathrm{b}}$ & 240 & $83 \mathrm{~d}$ & $82 \mathrm{~d}$ & 77 de & $105 \mathrm{e}$ & $241 \mathrm{de}$ \\
\hline Bentazon & 1080 & $75 \mathrm{~cd}$ & $45 \mathrm{~cd}$ & $58 \mathrm{~cd}$ & $83 \mathrm{~cd}$ & $221 \mathrm{cde}$ \\
\hline Thifensulfuron-methyl ${ }^{\mathrm{c}}$ & 6 & $73 \mathrm{~cd}$ & $77 \mathrm{~d}$ & $58 \mathrm{~cd}$ & $77 \mathrm{c}$ & $164 \mathrm{c}$ \\
\hline Chlorimuron-ethyl ${ }^{\mathrm{de}}$ & 9 & $18 \mathrm{~b}$ & $26 \mathrm{bc}$ & $14 \mathrm{~b}$ & $20 \mathrm{~b}$ & $25 \mathrm{~b}$ \\
\hline Cloransulam-methyl $^{\mathrm{ef}}$ & 17.5 & $77 \mathrm{~cd}$ & $89 \mathrm{~d}$ & 66 cde & 90 cde & $238 \mathrm{de}$ \\
\hline Imazethapyr $^{\mathrm{ef}}$ & 100 & $75 \mathrm{~cd}$ & $54 \mathrm{~cd}$ & $90 \mathrm{e}$ & 95 de & 212 cde \\
\hline 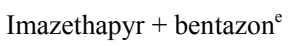 & $75+840$ & $57 \mathrm{c}$ & $65 \mathrm{~cd}$ & $78 \mathrm{de}$ & $78 \mathrm{c}$ & $174 \mathrm{~cd}$ \\
\hline Glyphosate & 900 & $12 \mathrm{~b}$ & $10 \mathrm{~b}$ & $48 \mathrm{~b}$ & $48 \mathrm{~b}$ & $8 \mathrm{~b}$ \\
\hline $\mathrm{SE}$ & & 3 & 4 & 4 & 4 & 11 \\
\hline
\end{tabular}

${ }^{\mathrm{a}}$ Means followed by the same letter within a column are not significantly different according to Fisher's Protected LSD at $P<0.05$; ${ }^{\mathrm{b}}$ Included Turbocharge $(0.5 \% \mathrm{v} / \mathrm{v}) ;{ }^{\mathrm{c}}$ Included non-ionic surfactant $(0.1 \% \mathrm{v} / \mathrm{v})$ and UAN $28 \%\left(8.0 \mathrm{~L} \cdot \mathrm{ha}^{-1}\right) ;{ }^{\mathrm{d}}$ Included non-ionic surfactant $(0.2 \% \mathrm{v} / \mathrm{v}) ;{ }^{\mathrm{e}}$ Included UAN $28 \%\left(2.0 \mathrm{~L} \cdot \mathrm{ha}^{-1}\right)$; ${ }^{\mathrm{f}}$ Included non-ionic surfactant $(0.25 \% \mathrm{v} / \mathrm{v})$.

only $30 \%$ control of Vigna unguiculata with acifluorfen at 4 WAA. Soltani et al. [18] concluded that fomesafen has an adequate margin of crop safety in adzuki bean, having no effect on plant height, dry weight, moisture or yield. Also, similar results are found in snap bean, where fomesafen applied POST at $0.84 \mathrm{~kg} \cdot \mathrm{ha}^{-1}$ resulted in only $13 \%$ injury at 6 WAA [13]. Bentazon applied POST to an adzuki bean crop caused $30 \%$ and $38 \%$ injury at 1080 and $2160 \mathrm{~g}$ ai.ha ${ }^{-1}$, respectively, 4 WAA, which is significant injury to a crop, but is inadequate efficacy for weed control [18]. Talbert et al. [13] reported that cloransulam-methyl has an adequate margin of crop safety in snap bean and southern pea for weed control in these crops. Cloransulam-methyl applied POST at $0.02 \mathrm{~kg} \cdot \mathrm{ha}^{-1}$, injured snap bean $29 \%$ and $15 \%$ at 6 and $8 \mathrm{WAE}$, while injury to southern pea was $24 \%, 18 \%$, and $18 \%$ at 5,8 , and 10 WAE, respectively. Talbert et al.'s [13] research in snapbean also documents imazethapyr $\left(0.03 \mathrm{~kg} \cdot \mathrm{ha}^{-1}\right)$ and bentazon $\left(0.84 \mathrm{~kg} \cdot \mathrm{ha}^{-1}\right)$ applied POST resulted in $25 \%$ and $10 \%$ injury at $6 \mathrm{WAE}$, respectively. Additionally, previous research shows that imazethapyr has an adequate margin of crop safety for application in adzuki bean [18].

Acifluorfen, fomesafen, bentazon, thifensulfuron-methyl, cloransulam-methyl, imazethapyr, and imazethapyr + bentazon applied POST did not reduce the number of volunteer adzuki bean plants with pods, seed production, or seeds per plant (Table 10). Also, the level of soybean contamination with adzuki bean seed was greater than the maximum allowable level of $1 \%$ and was not different than the weedy control. Based on this data the afore- mentioned POST herbicides do not provide adequate control of volunteer adzuki bean in soybean.

Chlorimuron-ethyl ( $9 \mathrm{~g}$ ai.ha $\left.{ }^{-1}\right)$ applied POST provided $45 \%$ to $90 \%$ control at 4 WAA and $66 \%$ to $84 \%$ control at 8 WAA, respectively. This level of control is consistent to that of Vigna unguiculata in a study by Jones et al. [16], where chlorimuron-ethyl applied POST at 8.8 g ai.ha ${ }^{-1}$ provided $95 \%$ and $93 \%$ Vigna unguiculata control at 4 WAA when sprayed 14 and 21 DAE, respectively. In this study, volunteer adzuki bean density and dry weight was also reduced by as much as $83 \%$ and $90 \%$, respectively. Plants with developed pods, seeds produced, and seeds per plant were all reduced compared to the weedy control. Soybean yield was greater with the application of chlorimuron-ethyl than the other herbicides tested for use in conventional soybean. Additionally, the soybean contamination with adzuki bean was below $1 \%$, which is acceptable for IP soybean.

Glyphosate (900 g ae.ha ${ }^{-1}$ ) applied POST provided $55 \%$ to $99 \%$ and $64 \%$ to $94 \%$ control at 4 and 8 WAA, respectively. Density and dry weight were reduced up to $84 \%$ and $97 \%$, respectively. Adzuki bean plants with pods, seed production, and seeds per plant were all reduced, while soybean yield equaled that of the weed free control and soybean contamination with adzuki bean seed was below $1 \%$.

\subsection{Volunteer Adzuki Bean Emergence}

Adzuki bean emergence data were collected in 2009 at the Ridgetown location. Figure 1 shows the cumulative 
Table 10. Means for volunteer adzuki bean growth in soybean with various POST herbicides ${ }^{\mathrm{a}}$. Data for plants with pods, seeds and seeds/plant obtained in 2009 at Ridgetown and Exeter, ON.

\begin{tabular}{|c|c|c|c|c|c|c|}
\hline \multirow{2}{*}{ Treatment } & \multirow{2}{*}{$\begin{array}{c}\text { Dose } \\
\text { g ai.ha }{ }^{-1}\end{array}$} & \multirow{2}{*}{$\begin{array}{l}\text { Plants with } \\
\text { pods } \# \mathrm{~m}^{2}\end{array}$} & \multirow{2}{*}{$\begin{array}{l}\text { Seeds } \\
\# \mathrm{~m}^{2}\end{array}$} & \multirow{2}{*}{$\begin{array}{c}\text { Soybean yield } \\
\mathrm{t} \cdot \mathrm{ha}^{-1}\end{array}$} & \multicolumn{2}{|c|}{ Contamination $\%$} \\
\hline & & & & & $2005 / 06$ & 2009 \\
\hline Untreated control & & $55 \mathrm{c}$ & $412 \mathrm{~b}$ & $2.28 \mathrm{~d}$ & $17 \mathrm{~cd}$ & $6 \mathrm{~b}$ \\
\hline Weed free control & & $0 \mathrm{a}$ & $0 \mathrm{a}$ & $3.03 \mathrm{a}$ & $0 \mathrm{a}$ & $0 \mathrm{a}$ \\
\hline Acifluorfen & 600 & $49 \mathrm{c}$ & $314 b$ & $2.21 \mathrm{~d}$ & $19 \mathrm{~cd}$ & $6 \mathrm{~b}$ \\
\hline Fomesafen $^{\mathrm{b}}$ & 240 & $60 \mathrm{c}$ & $417 \mathrm{~b}$ & $2.22 \mathrm{~d}$ & $20 \mathrm{~cd}$ & $6 \mathrm{~b}$ \\
\hline Bentazon & 1080 & $48 \mathrm{c}$ & $295 \mathrm{~b}$ & $2.49 \mathrm{bc}$ & $16 \mathrm{~cd}$ & $4 \mathrm{~b}$ \\
\hline Thifensulfuron-methyl $^{\mathrm{c}}$ & 6 & $45 \mathrm{c}$ & $215 b$ & $2.50 \mathrm{bc}$ & $11 \mathrm{c}$ & $4 \mathrm{~b}$ \\
\hline Chlorimuron-ethyl ${ }^{\mathrm{de}}$ & 9 & $6 \mathrm{~b}$ & $24 \mathrm{a}$ & $2.66 \mathrm{~b}$ & $2 \mathrm{~b}$ & $0 \mathrm{a}$ \\
\hline Cloransulam-methyl ${ }^{\text {ef }}$ & 17.5 & $55 \mathrm{c}$ & $382 \mathrm{~b}$ & $2.26 \mathrm{~d}$ & $22 \mathrm{~d}$ & $6 \mathrm{~b}$ \\
\hline Imazethapyr ${ }^{\text {ef }}$ & 100 & $56 \mathrm{c}$ & $331 \mathrm{~b}$ & $2.21 \mathrm{~d}$ & $19 \mathrm{~cd}$ & $7 \mathrm{~b}$ \\
\hline 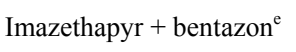 & $75+840$ & $56 \mathrm{c}$ & $317 \mathrm{~b}$ & $2.37 \mathrm{~cd}$ & $18 \mathrm{~cd}$ & $5 \mathrm{~b}$ \\
\hline Glyphosate & 900 & $13 \mathrm{~b}$ & $42 \mathrm{a}$ & $2.93 \mathrm{a}$ & $1 \mathrm{ab}$ & $0 \mathrm{a}$ \\
\hline $\mathrm{SE}$ & & 3 & 21 & 0.60 & 1 & 0.4 \\
\hline
\end{tabular}

${ }^{\mathrm{a}}$ Means followed by the same letter within a column are not significantly different according to Fisher's Protected LSD at $P<0.05$; ${ }^{\mathrm{b}}$ Included Turbocharge $(0.5 \% \mathrm{v} / \mathrm{v}) ;{ }^{\mathrm{c}}$ Included non-ionic surfactant $(0.1 \% \mathrm{v} / \mathrm{v})$ and UAN $28 \%\left(8.0 \cdot \mathrm{L} \cdot \mathrm{ha}^{-1}\right) ;{ }^{\mathrm{d}}$ Included non-ionic surfactant $(0.2 \% \mathrm{v} / \mathrm{v}) ;{ }^{\mathrm{e}}$ Included UAN $28 \%(2.0$ $\left.\mathrm{L} \cdot \mathrm{ha}^{-1}\right) ;{ }^{\mathrm{f}}$ Included non-ionic surfactant $(0.25 \% \mathrm{v} / \mathrm{v})$.

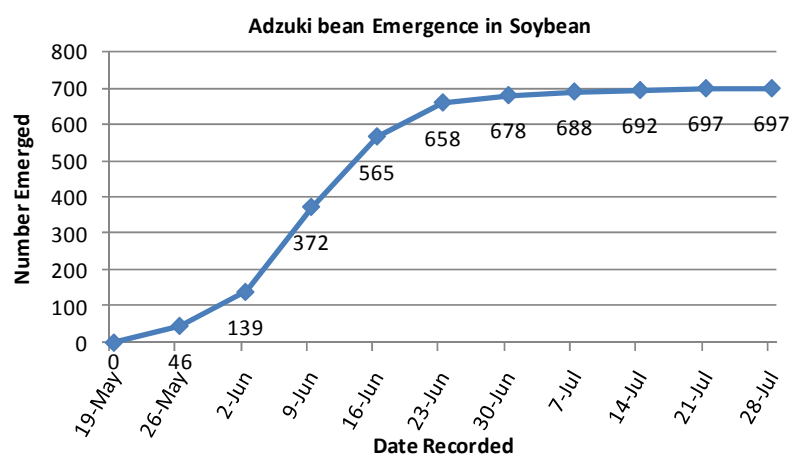

Figure 1. Cumulative emergence of volunteer adzuki bean in soybean at Ridgetown, ON in 2009.

emergence of volunteer adzuki bean within a soybean canopy. The soybean emerged on May 26 when $7 \%$ of the total adzuki bean population had emerged. By June 2, $20 \%$ of the total adzuki population had emerged. The majority of the population emerged in the following two weeks, as $61 \%$ of the total adzuki bean plants emerged between June 2 and June 16. At the time of the POST herbicide applications (June 24), 94\% of volunteer adzuki bean had emerged, meaning most of the population would have been exposed to the herbicide application. Volunteer adzuki bean is not as competitive in soybean since the adzuki bean emerges later than the soybean crop. However, as evidenced by the decreased yield when comparing the weedy to the weed-free control, the presence of volunteer adzuki bean reduced soybean yield, demonstrating a significant competitive effect.

\section{CONCLUSIONS}

None of the PRE herbicides evaluated provided acceptable control of volunteer adzuki bean in soybean. Of the PRE herbicides evaluated, linuron provided the best control of adzuki bean, as indicated by visual control ratings, and reduction in density and dry weight of volunteer adzuki bean. Most of the POST herbicides (acifluorfen, fomesafen, bentazon, cloransulam-methyl, imazethapyr, and imazethapyr plus bentazon) provided poor control of volunteer adzuki bean in soybean as indicated by visual control ratings and reductions in volunteer adzuki bean density and dry weight. Thifensulfuron-methyl applied POST provided higher levels of control, though not high enough to warrant consideration for use to control volunteer adzuki bean.

Chlorimuron-ethyl and glyphosate applied POST provided good control of volunteer adzuki bean at 4 and 8 WAA and reduced adzuki bean density and dry weight substantially. Additionally, chlorimuron-ethyl and glyphosate caused reductions in adzuki bean pod and seed production and resulted in soybean seed contamination with adzuki bean seeds of less than $1 \%$, protecting the premium price for an IP soybean grower.

In summary, the only herbicides evaluated in this study with potential to control volunteer adzuki bean are 
the POST application of chlorimuron-ethyl (9 $\mathrm{g}$ ai.ha $\left.{ }^{-1}\right)$ or glyphosate (900 $\mathrm{g}$ ae.ha $\mathrm{h}^{-1}$ ). All the other PRE and POST herbicides evaluated did not provide adequate control of volunteer adzuki bean in soybean.

\section{REFERENCES}

[1] McGill, J.A., Jr. (1995) Michigan-Japan and adzuki beans. Michigan Dry Bean Digest, 19, 4-7.

[2] Hensall District Co-operative (2010) Azuki bean production guide (F\#5B). Hensall District Co-operative, Ontario.

[3] Hardman, L.L., Oplinger, E.S. Doll, J.D. and Combs, S.M. (1989) Alternative field crops manual: Adzuki bean. University of Wisconsin-Cooperative Extension, Madison.

[4] Kaga, A., Isemura, T., Tomooka, N. and Vaughan, D.A. (2008) The genetics of domestication of the azuki bean (Vigna angularis). Genetics, 178, 1013-1036. doi:10.1534/genetics.107.078451

[5] Jansen, P.C.M. (2006) Vigna angularis (Willd.) Ohwi \& H. Ohashi. Record from Protabase. Brink, M. \& Belay, G. (Editors) PROTA, Wageningen, Netherlands. http://database.prota.org/search.htm

[6] Sacks, F.M. (1977) A literature review of Phaseolus angularis - The adsuki bean. Economic Botany, 31, 9-15. doi:10.1007/BF02860646

[7] Barrentine, W.L. (1974) Common cocklebur competition in soybeans. Weed Science, 22, 600-603.

[8] Weaver, S.E. (2001) Impact of lamb's quarters, common ragweed and green foxtail on yield of corn and soybean in Ontario. Canadian Journal of Plant Science, 81, 821828. doi:10.4141/P01-057

[9] Eaton, B.J., Russ, O.G. and Feltner, K.C. (1976) Competition of velvetleaf, prickly sida, and venice mallow in soybeans. Weed Science, 24, 224-228.

[10] Andersen, R.N., Ford, J.H. and Lueschen, W.E. (1982) Controlling volunteer corn (Zea mays) in soybeans (Glycine max) with diclofop and glyphosate. Weed Science, 30, 132-136.

[11] Beckett, T.H. and Stoller, E.W. (1988) Volunteer corn (Zea mays) interference in soybeans (Glycine max). Weed Science, 36, 159-166.

[12] Soltani, N., Shropshire, C. and Sikkema, P.H. (2006) Responses of various market classes of dry beans (Phaseolus vulgaris L.) to linuron. Weed Technology, 20, 118-122. doi:10.1614/WT-04-070R1.1

[13] Talbert, R.E., Schmidt, L.A., Lovelace, M.L. and Scherder, E.F. (1999) Field evaluation of herbicides on small fruit, vegetable, and ornamental crops, 1999. Arkansas Agricultural Research and Extension Center, Fayetteville.

[14] Harrison, H.F. (1988) Metribuzin tolerance within the genus Vigna. Weed Technology, 2, 59-63.

[15] Soltani, N., Shropshire, C., Robinson, D.E. and Sikkema, P.H. (2005) Sensitivity of adzuki bean (Vigna angularis) to preplant-incorporated herbicides. Weed Technology, 19, 897-901. doi:10.1614/WT-05-005R1.1

[16] Jones, J.D., Gossett, B.J., Harris, J.R. and Toler, J.E. (1989) Cowpea (Vigna unguiculata) control in soybean (Glycine max) with acifluorfen, chlorimuron, and thiameturon. Weed Science, 3, 518-522.

[17] Murphy, T.R. and Gossett, B.J. (1984) Control of cowpea (Vigna unguiculata) in soybean (Glycine max) with acifluorfen. Weed Science, 32, 427-431.

[18] Soltani, N., Robinson, D.E., Shropshire, C. and Sikkema, P.H. (2006) Adzuki bean (Vigna angularis) responses to post-emergence herbicides. Crop Protection, 25, 613-617. doi:10.1016/j.cropro.2005.09.013 